\section{Reverse translation: clearing a path from bedside to bench}

SIR - Your News Feature 'The Full Cycle' (Nature 453, 843-845; 2008), about taking research from bedside to bench, focused on unexpected results from clinical trials that stimulated a basic scientific investigation into what went wrong in those trials. Although this is a fascinating application of 'reverse translation', I fear it misses the much bigger opportunities and challenges that arise from researchers trying to understand clinical problems that are nowhere near the clinicaltrial stage. By listening to the problems experienced by our clinical colleagues, and having the resources to address those problems in novel and creative ways, researchers can not only contribute to patient benefit, but can also introduce new ways of thinking or whole new paradigms to basic research.

But there is a sting in the tail to this approach, mentioned in your News Feature 'Crossing the Valley of Death' (Nature 453, 840-842; 2008). Attempting to resolve apparently intractable clinical problems can be a valley of death for researchers who currently need to produce highimpact papers, reviewed mostly by basic scientists. The time taken to get to grips with a new problem, sometimes even a completely new approach, together with the necessarily less clear-cut experimental design inherent in clinical research, can become a 'do or die' endeavour for a biomedical scientist.

I believe the solution to this dilemma is procedural and cultural. Grant-review committees need to give a clear steer on the trade-off between many forms of clinical problem-solving and conventional criteria for scientific excellence. To this end, leaders of funding bodies must pay more than lip service to ensuring that both forward and reverse translation are placed at the centre of their funding strategy. Longer-term funding schemes, such as the intramural support provided by many public and private funding bodies on both sides of the Atlantic, can play a specific role in promoting risky but potentially fruitful translational research. In the end, though, all parties must somehow be persuaded to sign up to both health and research.

David R. Moore MRC Institute of Hearing Research, University Park, Nottingham NG7 2RD, and National Biomedical Research Unit in Hearing, Nottingham University Hospitals Trust, Nottingham NG7 2UH, UK

\section{Translational research: don't neglect basic science}

SIR - Recent editions of Nature have trumpeted the merits of translational research (Nature 453, 823, 830-831, 839-849; 2008) and then - almost as an afterthought to redress the balance - of basic research (Nature 453, 1144, 1150-1151; 2008). You highlight concerns that increased investment in translational research could be eroding support for basic research, evidence for which is writ loud on the UK Medical Research Council's web pages, for example. Of the many recent targeted calls for proposals, almost all are translational. As pointed out in Nature, this doesn't necessarily mean less money for basic research, but it does mean that basic science misses out on a share of the substantial amount of new money.

For investigators of basic science, this trend creates a conundrum. Go where the money is, or hope that basic science retains its financial foothold. Some adroitly plant a foot in each camp - although for many this is an unsatisfactory ploy, because basic and translational research have different challenges and rewards.

Basic science is typically hypothesis-driven and leads to new discoveries, whereas translational research applies those discoveries to patient benefits. The link between the two is exemplified by the discovery of small interfering RNA, which emerged from basic research that was eventually awarded the Nobel prize, and is now being tested as a therapeutic tool. The basic scientist may get as far as filing a patent application, but often loses interest in product development and commercialization.

Basic researchers should not have to worry about the validity of their work, but they will often add a rider to 'sell' their papers and grant applications - "these studies on [insert name of cell line or obscure gene] should pave the way for new treatments for [insert disease du jour]".

Such concerns underline the need to include champions of basic science among science policy-makers and the bodies that fund biomedical research. Otherwise the fear is not that basic science will be deliberately run down, but that it will indeed become an afterthought and die by neglect.

Stephen Moss Division of Cell Biology, Institute of Ophthalmology, University College London, 11-43 Bath Street, London EC1V 9EL, UK

\section{Health science: from bench to bedside to trench and back}

\section{SIR - The belated push to}

accelerate clinical discovery through translational research is an uncertain undertaking, as you remark in your Editorial 'To thwart disease, apply now' (Nature 453, 823; 2008).

In addition to the conversion of laboratory knowledge into new products and the adoption of such products by providers into routine clinical practice, clinical translational research must encompass behavioural and communications science in order

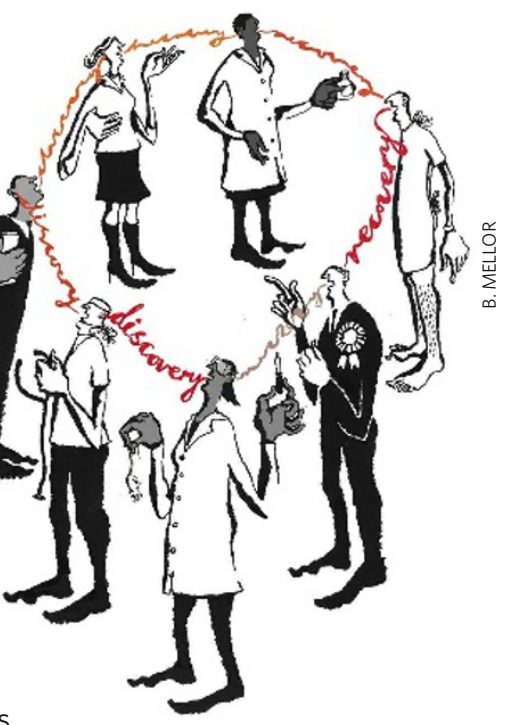

to study population uptake of new medical and scientific advances. This is crucial in a world of increasingly empowered, but not necessarily discerning, consumer patients suffering from information overload.

Community-based participatory research aims to engage the public, with a view to facilitating clinical-trial accrual and mobilizing the local expertise of community leaders. This can help to shape the investigations themselves, so that new science is both medically efficacious and effective in the real world of diverse people and patients.

The US National Institutes of Health (NIH) attempts to steer institutional culture and organizational ethics in this direction. It is supporting assessment of the effects of clinical translational science on social processes and infrastructure in academic medical centres, as well as the design of the research studies themselves.

Translational research should demonstrate a return on investment for basic science that is supported by the tax payer, and be a game-changing commitment by the NIH. The effort will need to transcend disciplinary silos to create a genuine delivery continuum for health science, from bench to bedside to trench and back again.

Simon J. Craddock Lee Department of Clinical Sciences, Division of Research Ethics, University of Texas Southwestern Medical Center at Dallas, 5323 Harry Hines Boulevard, Dallas, Texas 75390-9070, USA 